\title{
Le Miaoyuan guan 妙緣觀, temple des Affinités merveilleuses, une fondation taoïste des Ming à Pékin
}

\section{Marianne Bujard}

\begin{abstract}
The Miaoyuan guan in Peking was founded during the Ming dynasty by Chen Jin, a Vietnamese eunuch captured during the Chinese war against Vietnam in the beginning of the 15th century. After serving four emperors, Chen Jin, who became a Daoist devotee and an expert in the art of long life, reunited his own residence with an old Zhenwu temple next to it, restored the buildings, and founded the Miaoyuan guan dedicated to the Daoist cult. Three centuries later, the Miaoyuan guan became one of the capital residences of the chaplain of the Yongzheng emperor, Lou Jinyuan (1689-1776). Affiliated to the Way of the Heavently Masters, Lou Jinyuan was the abbot of the most important Daoist shrine of the Forbidden City, the Qin'andian and later of the Da Guangming dian and the Dongyue miao ; he was also put in charge of the Bureau of Daoist Registers, the Daolu si. The relation between Lou Jinyuan and the Miaoyuan guan is explained in an inscription written by the official, poet and literary critic Shen Deqian (1673-1769) who, in the same inscription recounts briefly the history of the temple. The article attempts to show, through a particular case, the constant involvement of the eunuchs, the priests and the scholars in the life of Peking's temples, until the 20th century, when the religious activities began to decline.
\end{abstract}

\section{Citer ce document / Cite this document :}

Bujard Marianne. Le Miaoyuan guan 妙緣觀, temple des Affinités merveilleuses, une fondation taoïste des Ming à Pékin. In: Cahiers d'Extrême-Asie, vol. 25, 2016. Vies taoïstes, communautés et lieux / Daoist Lives, Community and Place pp. 131-150;

doi : https://doi.org/10.3406/asie.2016.1474

https://www.persee.fr/doc/asie_0766-1177_2016_num_25_1_1474

Fichier pdf généré le 08/11/2019 


\title{
Le Miaoyuan guan 妙緣觀, TEMPLE Des Affinités Merveilleuses, UNE FONDATION TAOÏSTE DES Ming À PÉKIN
}

\author{
Marianne BUJARD
}

\begin{abstract}
The Miaoyuan guan in Peking was founded during the Ming dynasty by Chen Jin 陳謹, a Vietnamese eunuch captured during the Chinese war against Vietnam in the beginning of the Isth century. After serving four emperors, Chen Jin, who became a Daoist devotee and an expert in the art of long life, reunited bis own residence with an old Zhenwu temple next to it, restored the buildings, and founded the Miaoyuan guan dedicated to the Daoist cult. Three centuries later, the Miaoyuan guan became one of the capital residences of the chaplain of the Yongzheng emperor, Lou Jinyuan 婁近垣 (I689-1776). Affiliated to the Way of the Heavently Masters, Lou Jinyuan was the abbot of the most important Daoist shrine of the Forbidden City, the Qin'andian 欽安殿 and later of the Da Guangming dian 大光 明殿 and the Dongyue miao 東获廟; he was also put in charge of the Bureau of Daoist Registers, the Daolu si 道錄司. The relation between Lou Jinyuan and the Miaoyuan guan is explained in an inscription written by the official, poet and literary critic Shen Deqian 沈德潛 (I673-I769) who, in the same inscription recounts briefly the bistory of the temple. The article attempts to show, through a particular case, the constant involvement of the eunucbs, the priests and the scholars in the life of Peking's temples, until the zoth century, when the religious activities began to decline.
\end{abstract}

Dans la voie tracée par le professeur Kristofer Schipper qui lança en 1992 un programme d'étude des temples de Pékin et de leurs inscriptions commémoratives, appelé Pékin, ville sainte, une équipe franco-chinoise conduit depuis 2003 , avec le soutien de l'École française d'Extrême-Orient et de la Fondation Chiang Ching-kuo, un inventaire complet des temples situés dans la partie nord de Pékin, dénommée « ville tartare » depuis la conquête mandchoue. Ce programme, intitulé Épigraphie et mémoire orale des temples de Pékin - Histoire sociale d'une capitale d'empire, a réuni une documentation dont la publication finale comptera onze volumes comprenant plus de I 500 édifices et 640 inscriptions ${ }^{\mathrm{I}}$. À ce jour, quatre volumes ont paru et le cinquième est en cours ${ }^{2}$. Chaque temple y fait l'objet d'une notice rédigée à partir de

I. Voir Marianne Bujard et Ju Xi, «The Heritage of the Temples, a Heritage in Stone: An Overview of Beijing's Religious Epigraphy », China Perspectives, 2007, 4, p. 22-30 et Marianne Bujard, «Inventaire des temples de Pékin (1750-1949) : épigraphie, archives et enquêtes de terrain », Revue de l'bistoire des religions, 2010, 4, p. 663-682.

2. Marianne Bujard et Dong Xiaoping, éd., Beijing neicheng simiao beike zhi 北京內城寺廟碑 刻志, vol. I et 2, Pékin, Guojia tushuguan, 2oII ; Marianne Bujard, éd., Beijing neicheng simiao beike zhi 北京內城寺廟碑刻志, vol. 3, Pékin, Guojia tushuguan, 2013 ; Ju Xi 鞠熙, Guan Xiaojing 
sources diverses : la littérature, l'épigraphie, les archives impériales et républicaines et l'enquête sur place ; les estampages des inscriptions sont accompagnés d'une transcription qui en facilite la lecture et des photographies récentes ou anciennes sont reproduites. Cette documentation vise à mieux faire comprendre le fonctionnement de la société urbaine de Pékin à travers ses institutions religieuses et ses associations de culte.

L'étude qui suit n'est qu'une esquisse des possibilités offertes par cette documentation qui, pour être pleinement utile, doit encore être placée dans une base de données informatisée dont la réalisation est prévue.

\section{Un temple richement doté}

Le Miaoyuan guan 妙緣觀 ou temple des Affinités merveilleuses se trouvait au nord de la ville, non loin de la muraille, entouré de nombreuses autres fondations religieuses. Il comprenait au moment de son aménagement en ${ }^{4} 4{ }^{I}$ plusieurs cours et bâtiments 3 . On le voit représenté sur la carte de Pékin, dessinée en 1750 sur les ordres de l'empereur Qianlong 乾隆 (r. I736-I796) (fig. I).

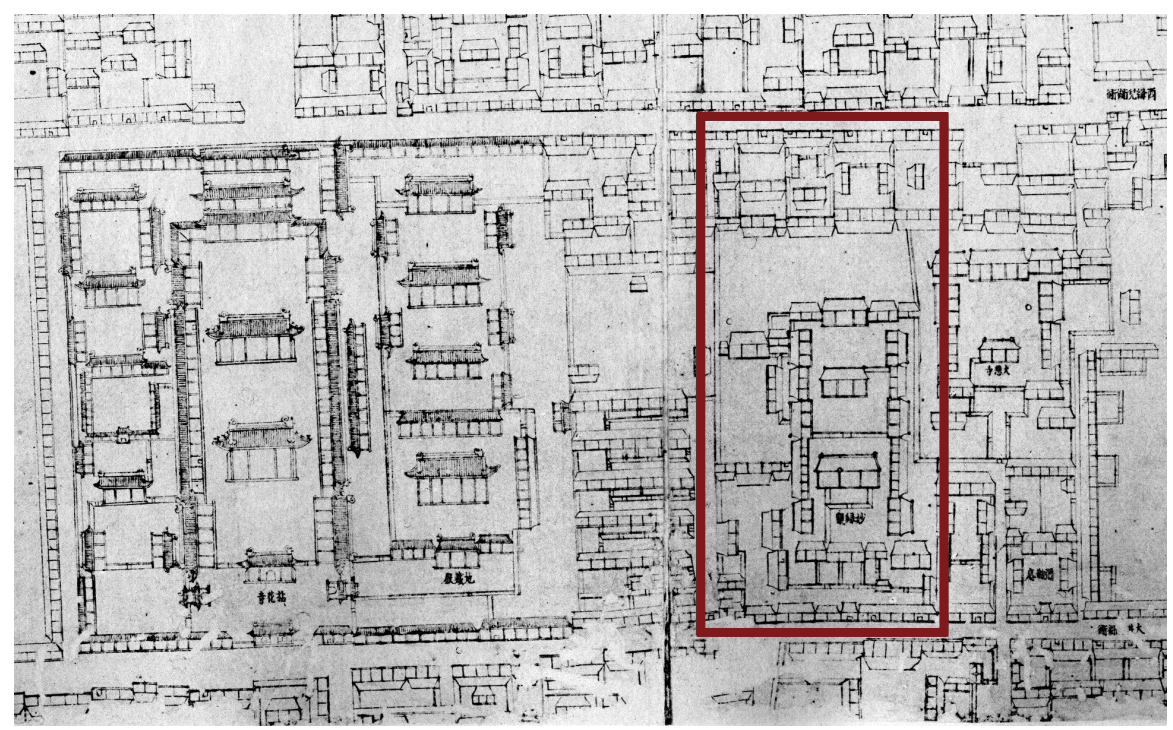

Fig. 1 : Dessin du Miaoyuan guan dans la Carte complète de la capitale 京城全圖 (ligne 1 , section 7, 一排七段 $)^{4}$.

關笑晶, Wang Minqing 王敏慶 et Lei Yang 雷陽, Beijing neicheng simiao beike zhi, Marianne Bujard, éd., vol. 4, 2 t., Pékin, Guojia tushuguan, 2017.

3. Voir la notice consacrée au Miaoyuan guan dans Beijing neicheng simiao beike zhi, vol. I, p. $182-203$.

4. Qing Neiwufu cang Jingcheng quantu 清内務府藏京城全圖, Gugong bowuyuan 故宮博物 院, Pékin, 1940. 
Sur cette carte, si l'on considère seulement les bâtiments qui se succèdent le long de l'axe central, ceux-ci comprennent près de 80 pièces'. D'après un cartulaire gravé en I45I, il faut encore y ajouter les cours disposées à l'est et à l'ouest du corps principal, si bien que le Miaoyuan guan comptait plus de 120 pièces $^{6}$ (fig. 2). Ce cartulaire gravé sur une stèle par les soins des deux prêtres administrateurs (zhuchi 住持) du temple $\mathrm{e}^{7}$ indique que le sanctuaire mesurait 40 zhang 丈 et 6 chi 尺 du nord au sud et i9 zhang et 5 chi d'est en ouest, soit environ $138 \mathrm{~m}$ de long sur $68 \mathrm{~m}$ de large ${ }^{8}$. Sans avoir les dimensions du grand monastère impérial bouddhique voisin, le Nianhuasi 拈花寺, il était plus vaste que la plupart des fondations religieuses de Pékin intra-muros 9 . En général, les temples comprenaient un portique d'entrée (shanmen 山門), une cour avec une salle principale et deux bâtiments latéraux de plusieurs pièces. L'ensemble était entouré par un mur d'enceinte. Lorsqu'il y avait une deuxième cour, le temple pouvait compter jusqu'à une vingtaine de pièces.

L'histoire de la fondation du Miaoyuan guan nous est rapportée par deux inscriptions, au contenu très semblable ${ }^{10}$, rédigées par le précepteur impérial et ministre des rites $\mathrm{Hu}$ Ying 胡淡 $\left(\mathrm{I} 375^{-\mathrm{I}} 463\right)^{\mathrm{II}}$. Elles livrent une description assez précise du lieu tel qu'il fut aménagé par son fondateur, l'eunuque Chen Jin 陳謹.

D'après ces inscriptions, le Miaoyuan guan réunissait les salles d'un ancien temple du Guerrier véritable Zhenwu 真武, le saint patron de la dynastie des Ming,

5. Nous traduisons par «pièce » le mot jian 間 qui désigne l'espace entre deux travées d'une construction.

6. L'inscription du cartulaire Chici Miaoyuan guan sizbi be $i$ 敕賜妙緣觀四至碑 a été transcrite par Ju Xi d'après l'estampage conservé à la bibliothèque de l'université de Pékin.

7. Li Chongqing 黎崇慶 et Li Chongli 李崇禮.

8. Les dimensions données dans l'une des stèles du Miaoyuan guan, datée de I45I, sont légèrement inférieures, voir Miaoyuan guan bei 妙緣觀碑, \# 539 (I45I), Beijing tushuguan cang Zhongguo lidai shike taben huibian 北京圖書館藏中國歷代石刻拓本匯編 (plus loin : BJTB), Pékin, Shumu wenxian, 1994, vol. 5I, p. 173.

9. Sur le Nianhua si, voir Beijing neicheng simiao beike zbi, vol. I, p. 207-253.

Io. Miaoyuan guan bei, \# 539 (I45I), BJTB, vol. 5I, p. 173, composée et calligraphiée par Hu Ying et Miaoyuan guan bei 妙緣觀碑, \# 538 (I452), BJTB, vol. 51, p. 178, composée par Hu Ying et calligraphiée par Cheng Nanyun 程南雲, fameux lettré et calligraphe. Le lecteur pourra se reporter à la reproduction des estampages de ces deux stèles, ainsi qu'à leur transcription, dans la disposition d'origine et en version ponctuée, dans Beijing neicheng simiao beike zbi, vol. I, p. I84-19o. Certaines des inscriptions présentées dans cet article ont été étudiées dans le cadre du groupe «Lecture de stèles de Pékin » qui s'est réuni mensuellement entre 2005 et 2006 à Paris et auquel ont assisté Isabelle Ang, Fang Ling 方玲, Vincent Goossaert, Pierre Marsone, Mao Chuanhui 毛傳慧, Shum Wim-fong 岑詠芳 et Pierre-Étienne Will. D’autres ont été traduites avec Victoire Surio. Patrice Fava a bien voulu relire cet article et m’a fait bénéficier de ses précieux conseils. J'ai aussi profité des remarques des éditeurs de ce volume. À tous, j'exprime ma vive reconnaissance.

II. Le BJTB, vol. 5 I reproduit dix-neuf inscriptions composées par Hu Ying entre I 438 et 1456. Susan Naquin parle d'au moins trente stèles rédigées par lui (Susan Naquin, Peking: Temples and City Life, I400-1900, Berkeley, University of California Press, 2000, p. 184). Le Daojia jinshi lüe 道家金石略 en comporte trois (p. 1254, I256, I259), dont deux ne se trouvent pas dans le BJTB, vol. 51. Cheng Nanyun, le calligraphe de la stèle de I452, a calligraphié des dizaines d'inscriptions, en particulier les caractères en sigillaire du cintre (bei'e 碑額). Hu Ying et Cheng Nanyun sont souvent associés dans l'épigraphie de l'époque. 

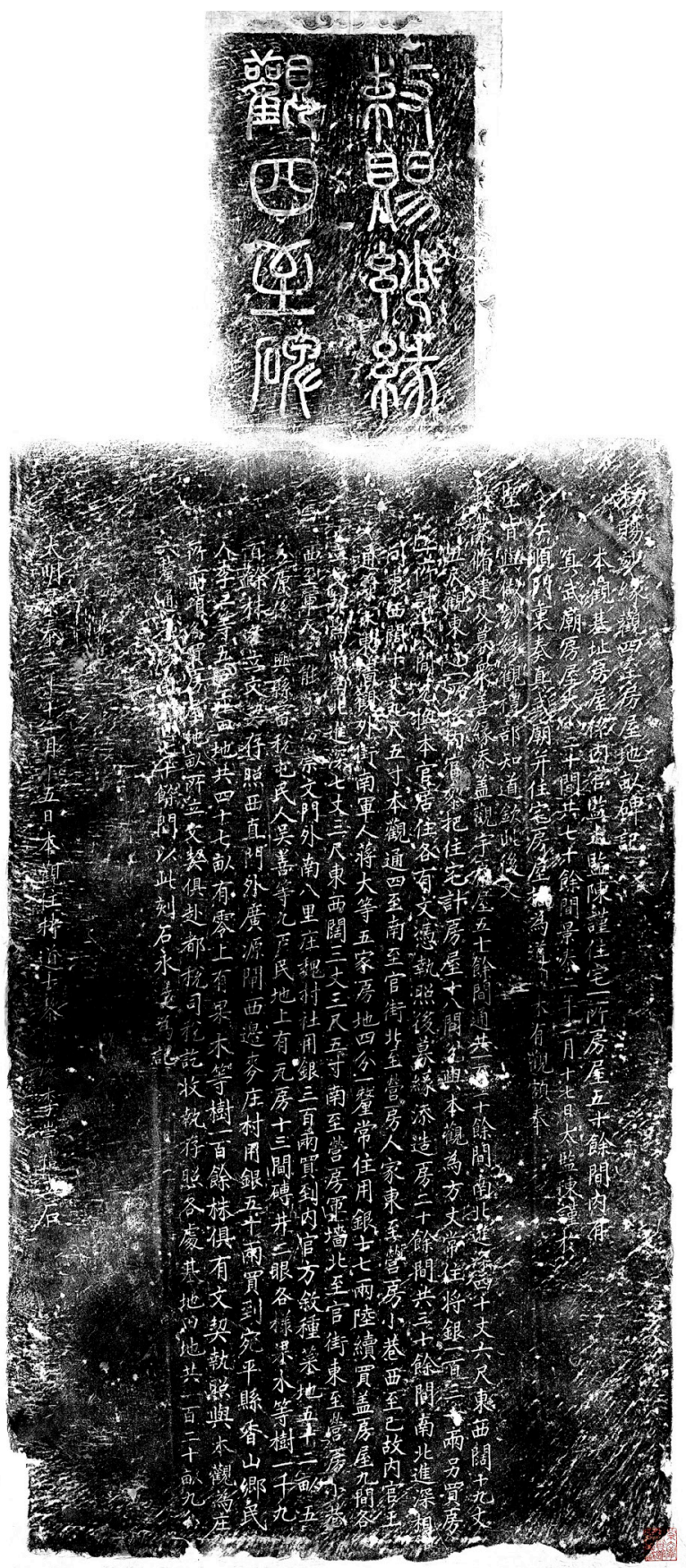

Fig. 2 : Le cartulaire du Miaoyuan guan ; estampage conservé à l'université de Pékin. 
composé d'une vingtaine de pièces grandes et petites, mais «trop exigu pour que l'on puisse y installer des statues $»^{\mathrm{I2}}$ et la résidence de l'eunuque qui comptait plus de cinquante pièces. Ajoutant aux subsides de la cour et aux aumônes des fidèles sa propre fortune, Chen Jin en agrandit encore l'espace pour y aménager un lieu de culte de plus de cent vingt pièces. Il acquit les dix-huit pièces de la cour située à l'est du sanctuaire, qui appartenait à un eunuque nommé Li Ba 黎把, afin d'y loger le supérieur du temple, et acheta pour I30 liang 兩 d'argent dix-huit autres pièces destinées aux eunuques résidents. Les inscriptions énumèrent le pavillon d'entrée shanmen, la tour de la Cloche et la tour du Tambour, la salle de la Grande Pureté (Taiqing dian 太清殿), celle du Tonnerre (Zhenming Lei dian 貞明雷殿), et, pour honorer Xuantian Shangdi 玄天上帝, le Guerrier sombre, alias Zhenwu 真武, la Chongzhen dian 崇真殿. Dans la salle Yinghua 應化殿 était déposé un exemplaire du Canon taoïste offert par l'empereur ${ }^{13}$. Le temple comprenait encore les cuisines, le réfectoire et les resserres, ainsi que les habitations des moines et du supérieur. «De part et d'autre du pavillon d'entrée couraient des galeries abritant des sculptures et des peintures représentant les maîtres parfaits et les généraux du passé, ainsi que les hauts faits des divinités et des immortels, et des vues de paysages réputés ${ }^{14}$.

La description physique des lieux évoque immédiatement celle d'autres temples de la même époque fondés eux aussi par des eunuques. Par exemple, deux ans plus tard, en I454, à quelques centaines de mètres du Miaoyuan guan, $\mathrm{Hu}$ Ying rédige une inscription pour le Qingxu guan 清虛觀 ou temple de la Pure Vacuitérs. Elle commémore pareillement la transformation de la résidence privée d'un eunuque en temple taoïste. En voici la description : «Lorsque les matériaux furent prêts, les travaux commencèrent par la salle située à l'avant ; on y honorait les seigneurs Dragon et Tigre (Longhu jun 龍虎君) ${ }^{16}$, ainsi qu'à gauche le véritable seigneur Wang et à droite le seigneur céleste $\mathrm{Liu}^{17}$. On construisit ensuite la salle centrale, où furent honorés les Trois Purs (Sanqing dian 三清殿), avec à gauche l'empereur du Ciel sombre (Zhenwu) et à droite le Vénérable céleste enseignant la doctrine

I2. Miaoyuan guan bei, \# 538 (I452), BJTB, vol. 51, p. I78.

13. La nouvelle édition du Canon taoïste fut gravée à l'initiative de l'empereur Yongle à partir des planches conservées au Baiyun guan ; Susan Naquin note qu'une copie y fut déposée et deux autres, au moins, offertes, l'une au Miaoyuan guan et l'autre à un temple de Zhenwu dans l'est de Pékin (voir Susan Naquin, op. cit., p. 95 et note 147).

I4. Miaoyuan guan bei, \# 538 (I452), BJTB, vol. 5 I, p. 178.

I5. Qingxu guan bei 清虛觀碑, \# 550 (I454), BJTB, vol. 5I, p. 190 ; voir aussi la notice consacrée au Qingxu guan, ainsi que la reproduction et la transcription de l'estampage de cette stèle dans Beijing neicheng simiao beike zhi, vol. 2, p. 602-6o6.

16. Le Dragon vert et le Tigre blanc, deux divinités gardiennes souvent postées à l'entrée des temples taoïstes.

17. Wang Zhenjun 汪真君 qui désigne Wang Zihua 汪子華 (714-789), disciple de Sima Chengzhen 司馬承禎, puis ermite au pic du Sud (Nanyue 南啍) révéré comme dieu du Feu 火師 dans le rituel des Cinq Tonnerres 五雷法; Liu Tianjun 劉天君 qui désigne Liu Hou 劉后 (317420), l'un des cinq généraux du ministère du Tonnerre (雷部五元師), à qui l'on s'adresse lors des rituels pour faire venir la pluie. 
(Yanjiao tianzun 演教天尊) ${ }^{18}$. À l'arrière, on éleva une haute salle à deux étages pour y prier l'empereur de Jade (Yuhuang 玉皇), flanqué à gauche du dieu du Sol (Tudi zhenguan 土地真官) et à droite des patriarches des générations antérieures (Lidai zushi 歷代祖師). Deux corps de bâtiments formaient les ailes tandis qu'un mur d'enceinte entourait l'ensemble ; un pavillon à trois portes ouvrait sur les tours de la Cloche et du Tambour. La salle de prière, la résidence de l'abbé, les cellules des moines, les resserres et les cuisines, tout était au complet et reflétait l'opulence de l'empire » (fig. 3).

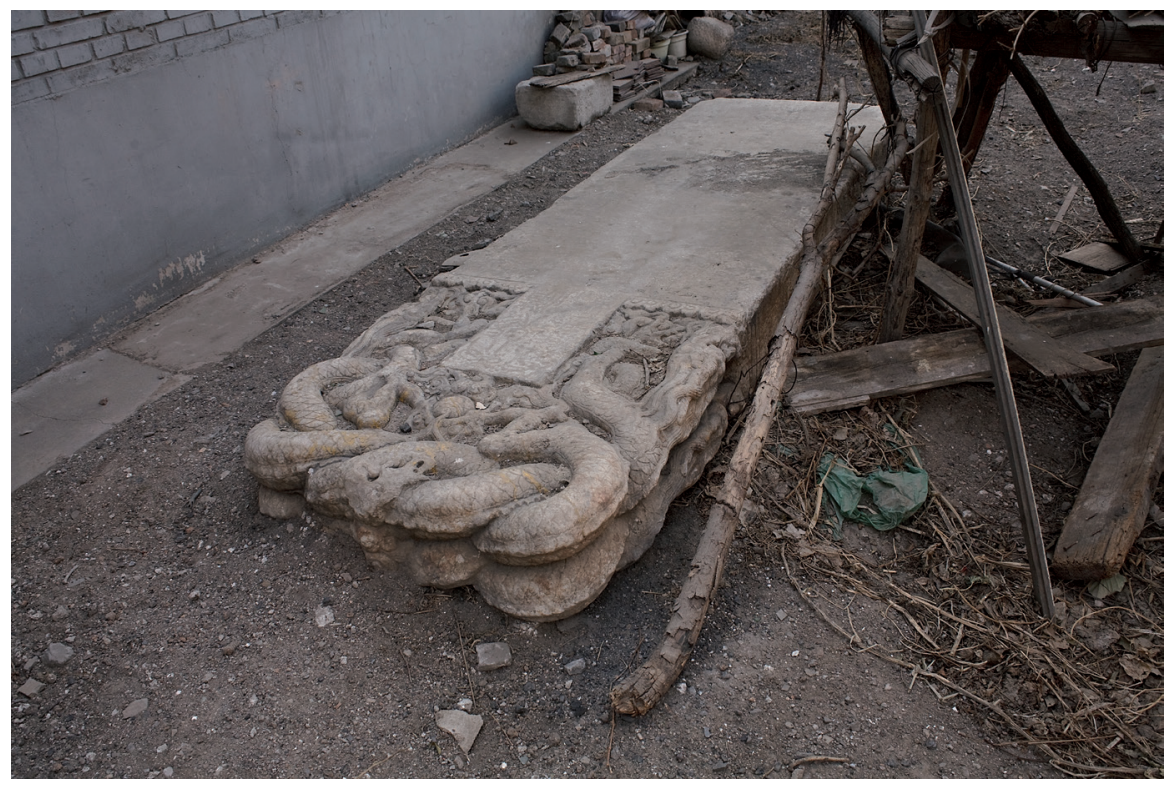

Fig. 3 : La stèle du Qingxu guan gravée en 1454, seul vestige in situ du temple aujourd'hui disparu. Photographie de David Gourhan, février 2012.

À cette époque, le pouvoir des eunuques était fermement établi. Tirant avantage de leur position auprès du souverain, ils contrôlaient la garde du palais impérial et la police secrète, tandis que par le biais des ateliers impériaux, dont ils assuraient la gestion, ils accumulaient des richesses considérables. Mais surtout, s'ajoutaient à leur fortune, les terres des environs de Pékin qu'ils obtinrent au titre de francs alleux. Ces terres étaient le plus souvent rattachées à des temples construits, restaurés ou agrandis par leurs soins. Peter Geiss a décrit le processus par lequel l'aristocratie impériale et les eunuques accaparèrent peu à peu les terres qui avaient été allouées aux colons et aux paysans au début de la dynastie : le plus souvent à tort, des terres étaient déclarées vacantes ; une demande d'octroi était alors engagée auprès de

I8. Sans doute Qiu Changchun (II48-I227), qui reçut de Kubilai en I269 le titre de maître de la Voie, Homme véritable enseignant la doctrine (長春主道演教真人). 
l'empereur pour le compte d'un noble ou d'un eunuque ; la demande satisfaite, les nouveaux acquéreurs en recevaient la propriété par privilège impérial. Les véritables propriétaires étaient expropriés et chassés, ou éventuellement embauchés comme ouvriers agricoles. Ces terres étaient dès lors exemptées d'impôt ${ }^{19}$.

Nombre de temples dans Pékin et ses environs furent édifiés par des eunuques sur les terres acquises par ce procédé. Selon les chiffres donnés par Susan Naquin, ces derniers fondèrent à Pékin et dans ses environs i40 temples, et en subventionnèrent $294^{20}$. Dans notre corpus, sur les 177 inscriptions datées de la dynastie des Ming et dédiées à des temples situés uniquement dans la ville intérieure, 62 commémorent des donations faites par des eunuques, soit plus d'un tiers. Privés de descendants, les eunuques étaient soucieux d'assurer le salut de leur âme par des œuvres pieuses. Reconstruire un temple, y installer des prêtres, les entretenir et faire célébrer des cultes en l'honneur de l'empereur, de la dynastie et de l'empire revenait à accumuler des mérites. En outre, le temple offrait un asile lorsqu'arrivait le moment de la retraite. Mais les temples étaient aussi le lieu de leur pouvoir temporel. Les eunuques y résidaient lorsque leurs affaires les amenaient à se déplacer et ils y rencontraient aussi bien des fonctionnaires que des membres du clergé et de l'aristocratie. Peter Geiss estime que les religieux jouaient alors le rôle d'intermédiaires entre les eunuques et les fonctionnaires qui briguaient leurs faveurs ${ }^{21}$.

Le cas du fondateur du Miaoyuan guan entre parfaitement dans ce schéma, aussi bien en ce qui concerne la fondation de temples en ville et à sa périphérie que pour l'acquisition de terres à la campagne. L'inscription de I45I évoque ainsi deux autres temples précédemment construits par Chen Jin, l'un dans l'est de la ville, le Xuanmiao guan 玄妙觀, dont on ne sait rien ${ }^{22}$, et le Lingying guan 靈應觀, dans les collines de l'Ouest (Xishan 西山) ${ }^{23}$. Pour ces deux fondations taö̈stes dédiées à Zhenwu, Hu Ying composa également des inscriptions ${ }^{24}$. On peut encore ajouter un autre sanctuaire taoïste, le Qingshui guan 清水觀 (aussi appelé Miaoqing guan 妙清觀), tout à l'ouest de la ville, près du mur d'enceinte. La stèle qui s'y trouve est datée de 1456 et a été aussi composée par Hu Ying ${ }^{25}$. Elle rapporte qu'en I445,

19. Geiss note qu'à la fin du $\mathrm{xv}^{\mathrm{e}}$ siècle, un sixième des terres imposables des environs de Pékin avait été accaparé par l'aristocratie et les eunuques, et échappait dès lors à l'impôt (Peter Geiss, "Peking under the Ming (1368-1644) ", thèse de doctorat, Princeton University, I979, p. IOI, voir aussi p. 90).

20. Susan Naquin, Peking, p. I80.

2I. Geiss, "Peking under the Ming », p. 98.

22. Il existe une inscription calligraphiée par Zhao Mengfu 趙孟煩 (I254-I322) datée des Yuan qui célèbre l'agrandissement de l'entrée d'un Xuanmiao guan mais je n'ai pu déterminer s'il s'agit du même temple qui aurait ensuite été restauré par Chen Jin (Xuanmiao guan sanmen ji 玄妙 觀三門記, \# 937I (sans date), BJTB, vol. 50, p. 150).

23. C'est dans les Xiangshan ou Collines Parfumées (qui font partie des collines de l'Ouest) que fut retrouvée la stèle funéraire de Chen Jin ; l'épitaphe indique que l'eunuque fit aménager sa tombe derrière le Lingying guan (Chen Jin muzbi 陳謹墓誌, \# 3962 (I479), BJTB, vol. 52, p. I45).

24. Je ne les ai pas identifiées.

25. Voir Miaoqing guan bei 妙清觀碑, BJTB, \# 357 (I456), vol. 51, p. 198 ; pour la transcription de cette stèle et la notice au sujet du temple, voir Beijing neicheng simiao beike zhi, vol. 3, p. 429-436. 
l'eunuque Chen Rixin 陳日新, affilié au département des Regalia (Sishe jian 司設監), transforma sa demeure en un temple pour Zhenwu ${ }^{26}$. Il en fit une dépendance (xiayuan 下院) du Wuhua guan 五華觀, un sanctuaire fondé par « l'Homme véritable Shao Yizheng 》 真人邵以正 (?-I463), haut dignitaire du bureau du Clergé taö̈ste (Daolu si 道錄司 $)^{27}$. Ces dépendances étaient nombreuses à la capitale. Elles servaient de lieu de résidence aux religieux attachés à un monastère dans les environs de Pékin lorsqu'ils se rendaient en ville pour traiter leurs affaires, prêcher ou rencontrer des fidèles. L'inscription rapporte que le nouveau temple de Zhenwu était exigu et sans attrait. On fit appel à Chen Jin, dont la piété et la générosité étaient connues, comme l'était la faveur dont il jouissait auprès de l'empereur. De fait, en I455, le Qingshui guan fut réparé et agrandi aux frais de la cassette impériale. À n'en pas douter, Chen Jin fut un promoteur très influent du taoïsme à Pékin. Son nom demeure attaché à plusieurs fondations, que le ministre des Rites, Hu Ying, ne dédaigna pas de commémorer dans sa prose élégante et sa calligraphie soignée ${ }^{28}$.

Le cartulaire gravé fait d'ailleurs état de plusieurs autres possessions attachées au Miaoyuan guan et acquises par Chen Jin. Il s'agit d'une part de maisons en ville, situées au sud du temple, de l'autre côté de la rue Dashiqiao 大石橋, qui appartenaient à des militaires et qu'il acquit pour 17 liang; il y fit construire neuf pièces d'habitation sur un terrain de plus de $200 \mathrm{~m}^{2}$. D'autre part, selon le procédé décrit par Peter Geiss, il acheta auprès d'un eunuque, douze $m u$ 畧 et demi de champs (deux hectares) au sud de Chongwen men 崇文門, dans le village de Bali zhuang 八里莊. Le cartulaire écrit que ces terres, acquises pour 300 liang par Chen Jin, appartenaient à l'origine à neuf familles de colons (tunmin 屯民); elles comprenaient treize maisons à toits de tuiles (wafang 瓦房), deux puits en briques et plus de 1900 arbres fruitiers de toutes espèces. En plus, Chen Jin acquit pour 50 liang, au-delà de la porte Xizhi 西直門, un autre domaine de $47 m u$ (près de 8 hectares) de terres agricoles, comprenant plus de cent arbres fruitiers, précédemment possédées par cinq familles de Xiangshan 香山 dans le district Wanping 宛平. L'inscription précise que tous les biens acquis, mobiliers et fonciers, furent déclarés au bureau

26. Le caractère $r i$ 日 dans le nom de l'eunuque indique peut-être son affiliation au lignage Changchun pai 長春派, fondé par Liu Yuanran. Shao Yizheng, qui eut pour nom religieux (faming 法名) Riyun 日雲, appartint pour un temps à ce lignage dont le poème comportait le caractère $r i$; voir Richard G. Wang, «Liu Yuanran and Daoist Lineages in the Ming », Daoism: Religion, History and Society, $\mathrm{n}^{\circ} 7,2015$, p. 286-287.

27. Sur Shao Yizheng, originaire du Yunnan et éminent disciple de Liu Yuanran 劉淵然 (I35I-I432), le fondateur du lignage Changchun 長春派, voir les quelques lignes dans l'Histoire des Ming, Mingshi 明史, «Fangji » 方伎, p. 7656, et une biographie plus complète dans l'inscription de I476 composée pour l'installation d'une chapelle dédiée à sa mémoire dans le Longquan guan 龍泉觀 près de Kunming (Longquan guan Tongmiao zhenren citang ji 龍泉觀通妙真人祠堂記, Daojia jinshi lüe, p. I265-I266). On y apprend notamment que Shao Yizheng fut enterré "par ordre impérial » au sud des montagnes Wuhua à l'ouest de Pékin, où se trouvait probablement aussi le Wuhua guan (敕葬京城西五華山之陽).

28. Hu Ying dit avoir fait la connaissance de Chen Jin pendant l'ère Yongle (I403-I425), alors qu'il était inspecteur en chef des Six Bureaux, chargé de la surveillance des Six Ministères et avait à ce titre l'occasion de rencontrer l'eunuque au service de l'empereur. 
des Impôts de la capitale, le Dushui si 都稅司, les taxes en furent acquittées et les reçus soigneusement conservés. Au total, c'est donc près de I23 mu (20 hectares) et plus de I70 pièces qui constituèrent les propriétés du temple et servirent autant à l'entretien des religieux qu'à celui des bâtiments et du mobilier rituel que l'on devine de précieuse facture.

\section{Un eunuque de royale ascendance}

Qui donc était Chen Jin ? Les deux inscriptions rédigées par le ministre des Rites fournissent à son sujet des éléments biographiques, que complète utilement son épitaphe. L'inscription de 1452 rapporte que " le sieur Chen est un adepte de l'Empereur jaune et de Laozi ; son surnom est Songgu 松谷 (vallée aux Pins) ${ }^{29}$. Il est né au Vietnam dans la maison royale des Tran (Chen) 陳. À la cinquième année de l'ère Yongle (I407), [le Vietnam] se soumit à la cour de Chine et [Chen Jin], se distinguant par ses talents et sa beauté, fut choisi pour entrer au palais en tant qu'eunuque ; il aimait réciter le Livre des Odes et le Livre des Documents " $^{30}$ Son ascendance royale, poursuit l'inscription, lui valut la protection de l'empereur Yongle (r. I4O2-I424). Séduit par ses qualités, le souverain l'employa dans les affaires du département des Eunuques du palais (Neiguan jian 內官監) ${ }^{31}$. L'épitaphe, rédigée à partir de sa fiche biographique (zbuang 狀), est plus précise : elle écrit que Yongle, après avoir évalué ses capacités, lui confia l'inspection du bureau des Constructions où Chen Jin s'illustra par sa compétence et son humanité envers les corvéables. Il

29. L'inscription de I45I ajoute que son nom personnel zi est Yunqing 雲卿 ; elle précise qu'il était un descendant des Yueshang 越裳, soit une façon littéraire de désigner son origine étrangère. Le terme désigne une population méridionale de la Chine ; la tradition veut que les Yuechang aient prêté allégeance au duc de Zhou en offrant en tribut un faisan blanc, voir Hanshi waizhuan 韓詩外傳 (Han Wei congshu 漢魏叢書, Changchun, Jilin daxue, 1992), juan 5, p. 47c. Les historiens vietnamiens les tiennent pour les anciens habitants de la région où s'installera dans les siècles suivants le royaume du Champa.

30. Chen Jin fit partie de la cohorte de jeunes gens au physique agréable et de bonne famille, choisis par le général Zhang Fu 張輔 (I375-I449) pour entrer au service de l'empereur en tant qu'eunuques. Parmi d'autres cas semblables, celui de Ruan An 阮安 (I38I-I453) est mieux connu ; il participa en tant qu'architecte à la construction de la Cité interdite et à plusieurs ouvrages hydrauliques (voir Mingshi 明史, juan 220, p. 777I). Je remercie Philippe Papin qui m’a renseignée à ce sujet.

3I. Le Neiguan jian était l'un des douze départements de l'administration des eunuques pendant la dynastie des Ming. Il avait en charge la fabrication du mobilier palatial et possédait plusieurs ateliers dans le quartier situé au nord-ouest de la colline de Charbon. Yu Minzhong 于敏中 dans son Rixia jiuwenkao 日下舊聞考 note qu'on y trouvait « les entrepôts de matériaux de construction de la porte Huangwa 黃瓦門, des greniers, des dépôts de sel, des ateliers de laqueurs, de ferronniers et de confection pour les mariages (bunlizuo 婚禮作), les marchés de l'est et de l'ouest, des tuiliers, des tailleurs de pierre, des potiers, des charpentiers, des dépôts de fournisseurs, des magasins, des ateliers de doreurs, des rizières, des ruches, des horticulteurs, des selliers, une manufacture de tuiles vernissées et des glacières ", voir Yu Minzhong, Rixia jiuwenkao (Pékin, Beijing guji, 2000, 4 vol.), vol. 3, p. 637 . 
servit ensuite l'empereur Hongxi 洪熙 (r. I424-I425) avec efficacité et docilité. Le souverain satisfait de la dévotion et de la sincérité avec lesquelles l'eunuque servait le Dao, lui décerna le titre de «diacre » (zhishi 執事) et le promut intendant du palais au deuxième échelon et spécialement affecté à l'autel Zixu yuxiu 紫虛根秀壇, où il priait matin et soir ${ }^{32}$. Il rejoignait ainsi les effectifs des eunuques qui secondaient les prêtres dans la célébration des cultes et des sacrifices impériaux. À son tour, Xuande 宣德 (r. I426-I435) le promut au poste d'archiviste, et lui accorda suffisamment d'estime pour lui confier la construction des deux sanctuaires taoïstes évoqués plus haut, le Xuanmiao guan et le Lingying guan. Ses bons et loyaux services valurent à Chen Jin une nouvelle promotion : il devint vice-directeur de gauche des eunuques (Zuoshao jian 左少監). Il demeura à ce poste jusqu'en I450, année où, du fait de son âge et de son ancienneté33 , le souverain Jingtai 景泰 (r. I449-I457) le nomma directeur du département des Eunuques du palais (Neiguan jian taijian 內官監太監).

L'épitaphe de Chen Jin, rédigée par un poète et lettré de l'Académie Hanlin 翰林院, Li Yongtong 李永通, a été retrouvée, on l'a vu, dans les collines Parfumées, derrière le temple Lingying, où de son vivant il avait pris soin d'aménager sa dernière demeure. Son commanditaire était l'administrateur du Miaoyuan guan, Wu Xuanhai 吳玄海, qui occupa les postes de «surintendant des cinq notes du bureau de la Musique » (Shenyue guan wuyin dutidian 神樂觀五音都提點) et de « Prédicateur de droite » (you yanfa 右演法), l'une des trois plus hautes fonctions du bureau de l'Administration du clergé taoïste ${ }^{34}$. L'inscription rapporte que Chen Jin mourut chez lui de maladie, durant l'été I479, à l'âge de 90 ans. Elle reprend les principales étapes de sa vie telles qu'elles ont été exposées dans les inscriptions rédigées par $\mathrm{Hu}$ Ying, mais s'attarde davantage sur les faveurs nombreuses que l'eunuque reçut des souverains. Ainsi, lorsqu'il fut promu directeur des eunuques en I450, l'empereur le gratifia de la ceinture de jade et du manteau brodé de motifs de dragons (yudai manglong fushi 玉帶蟒龍服飾), récompense dont seuls jouissaient les plus fidèles sujets. Durant l'ère Tianshun 天順 (I457-I464), le souverain lui offrit à plusieurs reprises des statues de Zhenwu, Wenchang 文昌 (Zitong), Lingguan (Wang Lingguang 王靈官 ?) et Guandi 關帝 afin qu'il leur rende un culte dans les temples qu'il avait fondés (累荷 賜以真武梓潼靈官關師神像). S’y ajoutèrent de l'ivoire et des habits brodés. À son tour, Chenghua 成化 (r. I464-I487), en reconnaissance de l'indéfectible dévouement de l'eunuque, lui offrit un nouveau manteau brodé de motifs de dragons. Il s'assura de son confort en lui faisant régulièrement porter des lingots d'or prélevés sur la cassette impériale. L'épitaphe se termine comme il se doit par un éloge des vertus du défunt qui répète tout ce qui précède en vers quadrisyllabiques.

32. 賜其正教執事, 陡爲長隨內侍, 專於紫虛欎秀壇, 晨夕焚修. Cet autel n'a pas encore été identifié.

33. D'après son épitaphe, Chen Jin était né le $5^{\mathrm{e}}$ jour du $4^{\mathrm{e}}$ mois lunaire de l'an $\mathrm{I} 39 \mathrm{O}$. Il avait donc 6I ans en 1450 dans la façon chinoise de compter.

34. Sur le bureau de la Musique, voir Liu Yonghua, «Daoist Priests and Imperial Sacrifices in Late Imperial China: The Case of the Imperial Music Office (Shenyue Guan), I379-1743 ", Late imperial China, vol. 33, $\mathrm{n}^{\circ}$ I (2012), p. 55-88. 


\section{Un eunuque vietnamien en milieu taoïste}

L'inscription du Miaoyuan guan de 1452 rapporte que lorsque Chen Jin demanda à l'empereur l'autorisation de transformer sa demeure en temple taoïste, il reçut non seulement une aide financière pour mener à bien les travaux de transformation, mais encore un exemplaire du Canon taoïste Daozang zhenjing 道藏真經. Celui-ci fut déposé dans une salle spécialement construite à cet usage.

On sait que la compilation du nouveau Canon taoïste revint d'abord à Zhang Yuchu 張宇初 (I36I-I4IO), le $43^{\text {e }}$ Maître céleste ${ }^{35}$. À sa mort en I4IO, Zhang Yuqing 張宇清, son frère cadet et successeur, poursuivit le travail d'édition ; en I444, c'est Shao Yizheng qui corrigea les épreuves et supervisa l'impression en I445. Mais Zhang Yuchu fut aussi l'éditeur des écrits du Véritable Seigneur de la paisible vacuité (Xujing zhenjun 虛靖真君), alias le trentième Maître céleste, Zhang Jixian 張繼先 (I092-II27) qu’il publia sous le titre «Enseignements du trentième Maître céleste Xujing Zhenren »三十代天師虛靖真君語錄. Le Miaoyuan guan abrite une stèle non datée, mais calligraphiée par Chen Jin lui-même, qui recopie le «Da Dao ge » 大道歌 ou " Chant du grand Dao », un texte inclus dans l'ouvrage de Zhang Jixian ${ }^{36}$. Sa reproduction sur une stèle témoigne de sa circulation au milieu du $\mathrm{XV}^{\mathrm{e}}$ siècle, quelque quarante ans après la mort de son éditeur. Cette stèle porte sur son envers une inscription intitulée «Stèle en cent caractères de l'immortel (Lü) Dongbin pour préserver et nourrir le champ du cœur» (洞賓仙人保養心田百字碑), laquelle décrit en vingt phrases de cinq caractères, une méthode fondée sur la méditation et l'alchimie intérieure « pour monter au ciel» (shang tianti 上天梯), c'est-à-dire devenir immortel ou, en tout cas, prolonger ses jours ${ }^{37}$. Les éditeurs du catalogue des estampages des inscriptions de Pékin conservées à la Bibliothèque nationale de Chine donnent pour calligraphe de cette inscription un certain Chongxuan ting 冲玄亭 ${ }^{8}$, à moins que ces trois caractères ne désignent le pavillon où le calligraphe a tracé son texte.

Chen Jin évolua dans un milieu d'eunuques, adeptes comme lui du taoïsme et suffisamment aisés pour patronner des fondations religieuses. Certains d'entre eux furent ordonnés et firent fonction de prêtre dans leur propre demeure transformée

35. Sur cet éminent personnage, voir la contribution de Vincent Goossaert dans ce volume: 《The Four Lives of Zhang Yuchu 張宇初 (I36I-I4IO), 43rd Heavenly Master ».

36. Xujing zhenjun dadaoge 虛靖真君大道歌, BJTB, \#54I (sans date), vol. 60, p. 177. Chen Jin se désigne sous son nom honorifique Songgu, précédé d'un autre nom, Guolong 郭龍, en plus petits caractères, sans doute un nom de lieu que je n'ai pas identifié. L'estampage de cette stèle et sa transcription se trouvent dans Beijing neicheng simiao beike zhi, vol.I, p. I9I-I96.

37. Dongbin xianren baoyang xintian baizi bei 洞賓仙人保養心田百字碑, BJTB, \# 542 (sans date), vol. 6o, p. 156 .

38. Beijing tushuguan cang Beijing shike tapian mulu 北京圖書館藏北京石刻拓片目錄, Pékin, Shumu wenxian, 1994, p. 5 II. Il pourrait s'agir du « pavillon de Chongxuan », Chongxuan désignant Shao Yizheng. Le caractère chong 冲 figure dans deux de ses titres, celui qui le désigne dans la stèle du Miaoqing guan : 守玄冲靖秉誠專確志道衍教妙悟靜虛弘濟真人, et celui qui lui fut conféré par l'empereur durant l'ère Jingtai (I45O-I456) : 悟玄養素凝神沖默闡微振法通妙真人. Le caractère xuan 玄 provient du lignage fondé par son maître Liu Yuanran. Sur ce personnage, voir Richard G. Wang, "Liu Yuanran and Daoist Lineages in the Ming ». 
en lieu de culte. Une étude systématique des inscriptions consacrées au patronage religieux des eunuques à Pékin reste à faire. Dans les cas de figure que nous avons rencontrés, nous observons d'une part, des restaurations telles que celle du Qingxu guan, qui ne concerne qu'un seul temple et son promoteur, aidé par quelques-uns de ses collègues ; et d'autre part, celle du Miaoyuan guan, qui englobe au moins quatre temples : celui qui fait l'objet de cette étude, le Miaoyuan guan, deux autres que Chen Jin a reconstruits, de lui-même ou mandaté par le souverain, l'un en ville, l'autre dans les collines de l'Ouest, le quatrième étant le Qingshui guan (ou Miaoqing guan), présenté comme une dépendance du Wuhua guan, le temple fondé par Shao Yizheng.

Davantage que la fondation du Qingxu guan, celle du Miaoyuan guan nous met en contact avec les élites de l'époque. Chen Jin, sans avoir jamais occupé les plus hautes fonctions dans l'administration du palais, fut en relation avec les grands personnages du clergé taoïste. Il était sans doute plus cultivé que la moyenne des eunuques, et sa foi taoïste était nourrie autant de lectures que de la connaissance des rituels. Son épitaphe qui répète les raisons pour lesquelles l'empereur Hongxi 洪熙 (I424-I425) l'avait délégué au service des rituels impériaux le présente comme un maître taoïste accompli : «L'empereur Zhao 昭 (Hongxi), dont le nom posthume est Renzong 仁宗, l'appréciait tout particulièrement ; comme le sieur [Chen Jin] était versé dans les enseignements de l'Empereur jaune et de Laozi et qu'il en maîtrisait les rituels, il le coiffa du bonnet en forme de lotus, le vêtit de l'habit pourpre (des prêtres taoïstes) et l'affecta au service des rituels jiao 醮.» L'autorité de Chen Jin en matière de recettes de longue vie, solidement établie par sa propre longévité, était telle que le souverain régnant fit quérir « les rituels taoïstes et les recettes secrètes de longue vie qu'il avait réunis dans le passé ». Le vieil eunuque les recopia un à un et l'empereur en fit grand $\operatorname{cas}^{39}$.

Parmi les temples fondés par les eunuques, celui des Affinités merveilleuses est singulier du fait qu'il fut construit par un eunuque d'origine vietnamienne. Cependant, la richesse du commanditaire, les hautes fonctions du rédacteur des inscriptions et le prestige du calligraphe, en font un exemple parfaitement représentatif du rôle que jouèrent les eunuques de la dynastie des Ming dans la construction des temples de Pékin et des liens privilégiés qu'ils entretenaient avec les élites de la capitale.

\section{Le Miaoyuan guan, une des résidences du chapelain de l'empereur Qianlong}

Lorsqu'on retrouve 300 ans plus tard, la trace du Miaoyuan guan dans l'épigraphie, c'est toujours une fondation taoïste. Une inscription de 1756 nous apprend que le temple est désormais une des résidences métropolitaines (xiayuan下院) du " grand chapelain » de Yongzheng 雍正 (r. 1723-1735), le prêtre taoïste Lou Jinyuan 婁近垣 $\left(\mathrm{I} 6899^{-1776}\right)^{40}$. Vincent Goossaert a dédié plusieurs pages à la vie de ce haut

39. Chen Jin muzhi, \# 3962 (I479), BJTB, vol. 52, p. I45.

40. Miaoyuan guan bei 妙緣觀碑, \# 543 (I756), BJTP, vol. 7I, p. 74 ; pour la transcription, voir Beijing neicheng simiao beike zhi, vol. I, p. 20I-203. 
dignitaire de l'Église des Maîtres célestes 天師道 ${ }^{4}$. Récemment, dans un article consacré aux relations entre les empereurs et les maîtres taoïstes, et dans lequel est reproduite une peinture représentant un rituel de guérison pour le frère de l'empereur Yongzheng, Fu You 蜉蝣 (alias Wang Zilin 王子林) pense pouvoir identifier Lou Jinyuan dans la personne du prêtre ${ }^{42}$. Véridique, cette identification confirmerait la grande faveur dont jouissait Lou Jinyuan auprès du souverain. Jusqu'à l'accession au trône de l'empereur Qianlong, qui rétrograda les Maîtres célestes 天師, ceux-ci se rendaient régulièrement en audience à la cour. C'est dans la suite du cinquantecinquième d'entre eux, Zhang Xilin 張錫麟, que Lou Jinyuan arriva à Pékin en $1727^{43}$. Il allait devenir le maître d'œuvre des grands rituels de la cour mandchoue et diriger à partir de 1740 le bureau de l'Administration du clergé taoïste. Apprécié par Yongzheng, il fut nommé en I73I responsable du Qin'an dian 欽安殿, le grand temple consacré à Zhenwu au nord de la Cité interdite ; il reçut deux ans plus tard le titre d'« Homme véritable », coiffé par l'épithète de "merveilleuse rectitude », Miaozheng zhenren 妙正真人. La même année, il devenait le premier administrateur du Da Guangming dian 大光明殿, le siège des rituels taoïstes réservés au souverain ; fondé sous les Ming, cet ensemble venait d'être entièrement restauré (fig. 4). Avec quarante-huit officiants faguan 法官, Lou Jinyuan y exécutait « les grands rites impériaux, les cérémonies pour demander ou faire cesser la pluie, les cultes pour assurer la paix de l'empire et protéger le peuple, les actions de grâce et les vœux de bonheur ${ }^{44}$. Il occupait encore cette charge en I770, comme l'indique la " liste des bâtiments des fondations religieuses subventionnées » (guanguan simiao dianyu fangiian shumu qingdan 官管寺廟殿宇房間數目清單), où son nom figure en tant qu'administrateur du Da Guangming dian ${ }^{45}$.

4I. Vincent Goossaert, «Bureaucratic Charisma: The Zhang Heavenly Master Institution and Court Taoists in Late-Qing China ", Asia Major, 3rd ser., vol. I7, n² 2, 2004, p. I2I-159, en particulier p. I4I-I49.

42. Fu You, «Daoshu xiandan changsheng meng: Diwang yu daoshi » 道術仙丹長生夢—— 帝王與道士, Zijincheng 紫禁城, 244, 2015, p. 36-45. La peinture est conservée à l'Arthur Sackler Gallery, Smithsonian Institution, Washington D.C. ; elle est reproduite et étudiée également dans Taoism and the Arts of China, Chicago, The Art Institute of Chigago, 2000, p.19o-I9r. Je remercie Patrice Fava qui m'a signalé ces articles. Patrice Fava suggère en outre que le portrait du maitre taoïste qui figure à la page 194 du catalogue de Chicago pourrait aussi représenter Lou Jinyuan (Portrait of a Taoist Priest, conservé au Royal Ontario Museum, Toronto, The George Crofts Collection).

43. Zhang Xilin mourut à Hangzhou et Lou Jinyuan continua le voyage sans lui.

44. Da Zhenren fu bei 大真人府碑, \# 549 (I740), BJTB, vol. 69, p. 64 ; pour la transcription, voir Beijing neicheng simiao beike zhi, vol. I, p. 197-200.

45. Ce registre appartient aux archives de la Maison impériale (Neiwufu 內務府) et comporte la liste des 72 temples subventionnés par l'État, voir Archives no I, dossier 05-0277-032. 

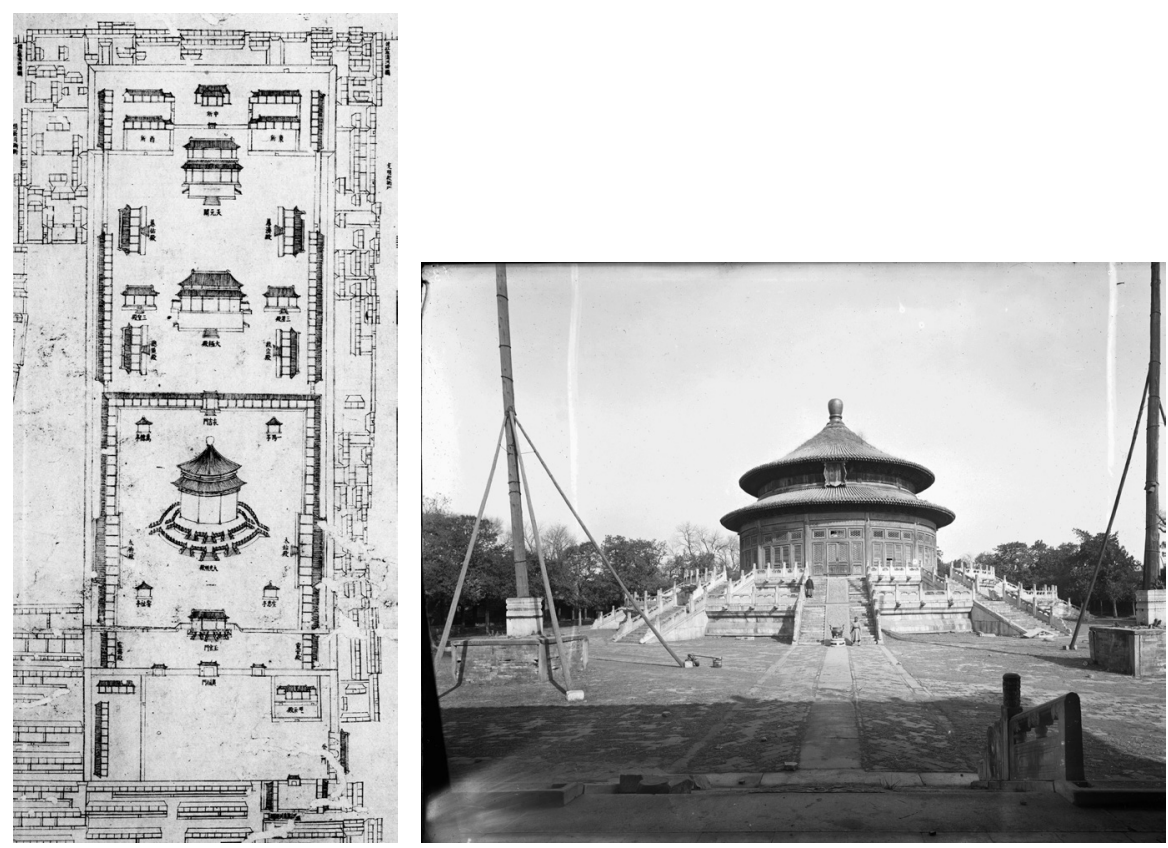

Fig. 4 : Dessin du Da Guangming dian dans la Carte complète de la capitale 京城全圖 (ligne 7, section 8, 七排八段) et photographie du temple central, Guangming dian, prise en 1892 par Charles Vapereau (Collection d'Arnaud Gibault, reproduite avec son aimable autorisation).

Ces affectations prestigieuses ne doivent pas faire oublier le strict contrôle que la bureaucratie impériale exerçait sur ses agents, fussent-ils des « hommes véritables ». Alors que Lou Jinyuan est en charge d'une autre grande fondation impériale taoïste, le Da Gaoxuan dian 大高玄殿, au pied de la colline de Charbon, à l'ouest de la Cité interdite ${ }^{46}$, les archives de la Maison impériale rapportent dans leur inimitable verve procédurière, un épisode fâcheux dans la carrière du " prélat » (fig. 5). Au cinquième mois de l'an 1755 , alors que Lou Jinyuan dirige un rituel dans le Da Gaoxuan dian, un prêtre de sa suite abîme un ustensile. Lou Jinyuan est aussitôt rabaissé de deux degrés (jiang er ji 降二级) ; par faveur impériale, la rétrogradation est ramenée à un degré. La requête conservée dans les archives introduit la demande de réhabilitation de Lou Jinyuan trois ans plus tard ${ }^{47}$.

Ces mêmes archives nous apprennent que Lou Jinyuan mourut de maladie à Pékin en 1776 et que sa dépouille fut escortée au Longhushan 龍虎山 (le siège de

46. Longtemps occupé par l'Armée populaire de Libération, ce temple est en cours de restauration et sera prochainement ouvert au public.

47. Archives n ${ }^{\circ}$, dossier 05-0166-002. 


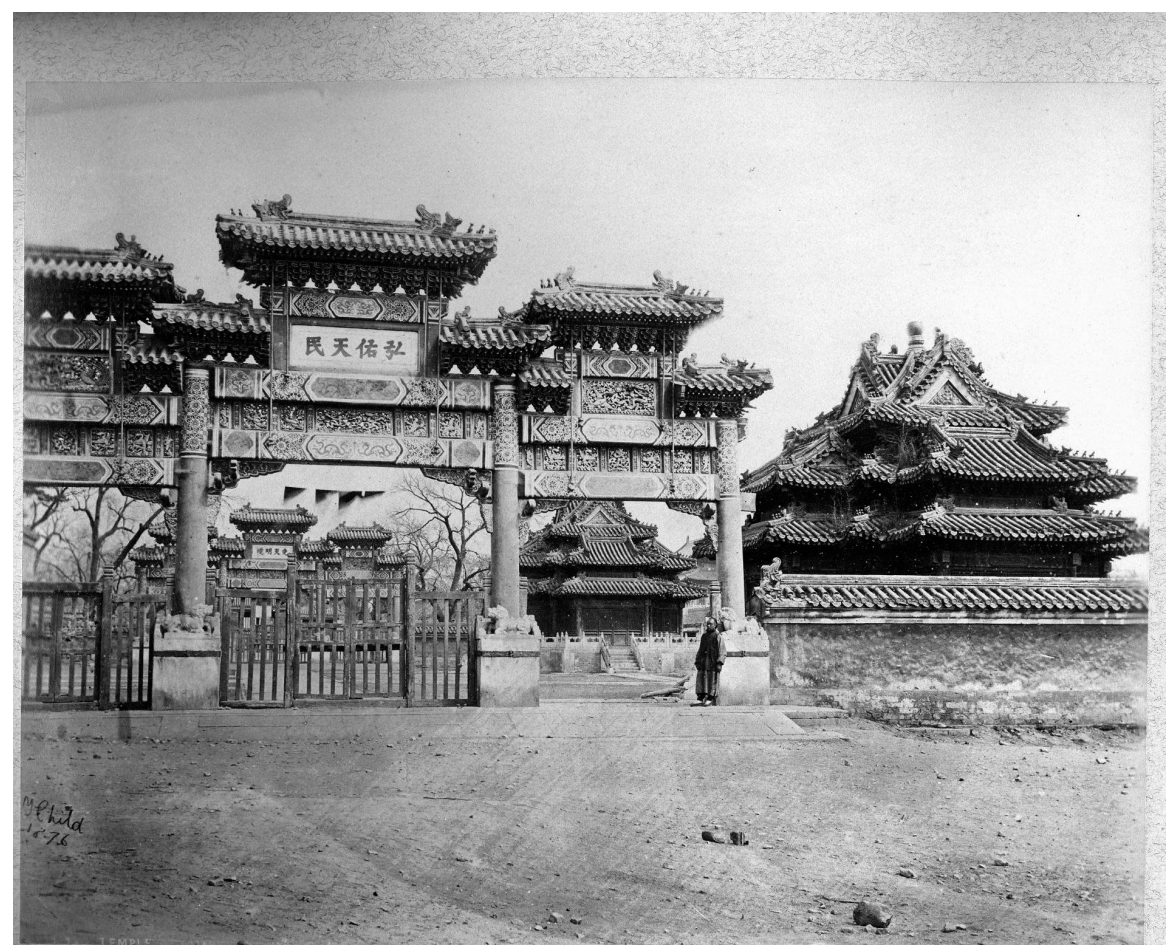

Fig. 5 : Photographie du portique occidental du Da Gaoxuan dian prise en 1860 par Felice Beato (Collection d'Arnaud Gibault, reproduite avec son aimable autorisation).

l'administration des Maîtres célestes) le $2^{\mathrm{e}}$ jour du $9^{\mathrm{e}}$ mois lunaire de l'an $\mathrm{I} 776$ par Zhan Ziguo 詹資國, un intendant (tidian 提點) du temple Shangqing du Longhushan ${ }^{48}$.

Lou Jinyuan est associé par le biais de deux inscriptions au Miaoyuan guan. La première datée de 1740 est en fait une stèle de commémoration de la « résidence de l'Homme véritable », le Zhenren fu 真人府. Elle est largement consacrée à la carrière de Lou Jinyuan, qui a atteint son apogée : à cinquante ans, à l'instar du Maître céleste, il porte le titre de zhenren, il a été nommé au troisième échelon sanpin 三品, avec la fonction honorifique de « gentilhomme de Bon Conseil » (Tongyi dafu 通議 大夫), il est à la tête du Da Guangming dian et d'autres temples parmi lesquels celui du pic de l'Est, le Dongyue miao 東獄廟 ${ }^{49}$; il a obtenu la restauration et

48. Archives $\mathrm{n}^{\circ} \mathrm{I}$, dossier 05-I422-0I9; ce document rapporte l'utilisation abusive du sceau de Lou Jinyuan et diverses fautes commises par des membres du bureau du Clergé taoïste. Voir aussi Qianlong shilu 乾隆實錄, juan IOI7 (http://m.xuehuile.com/blog/3605694a495I4brcbc3b8f66 I46cci39.html, consulté le 8 août 20I5) où le même Zhan Ziguo, arrivé au Longhushan, enfreint à nouveau la discipline.

49. Il rédigea deux inscriptions pour le temple du pic de l'Est, en I74I (BJTP \# IO26, vol. 69, p. 87-88) et 1756 (BJTP \# 930, vol. 7I, p. 85-87) ; voir aussi Beijing Dongyue miao yu Beijing 
l'embellissement des temples du Longhushan et dirige le bureau du Clergé taoïste. C'est alors que vient encore s'ajouter la jouissance du Zhenren fu, demeure ordinairement destinée au Maître céleste lorsqu'il se rend en audience à la cour (fig. 6). Autrefois construite au sud de la porte Zhengyang 正陽門, mais abandonnée car jugée trop exiguë, la nouvelle résidence est reconstruite entre I73I et I734 au sud-est de la tour du Tambour, au nord de Mao'er hutong 帽兒胡同, sur un terrain de près de $3000 \mathrm{~m}^{2}$. Le Maître céleste et sa suite y jouissaient de quatrevingt-et-une pièces, meublées par les ateliers impériaux et disposées au cœur d'un havre de paix et de verdure $^{50}$. Tout, nous dit l'inscription, y respirait le luxe et l'abondance ${ }^{\jmath 1}$.

Rédigée par le Maître céleste par intérim Zhang Zhaolin 張昭麟, cette stèle à la gloire de Lou Jinyuan dut être érigée d'abord dans le Zhenren fu. Lorsqu'en 1756 , le bureau de la Gendarmerie (Tidu fu 提督府) s'étendit jusqu'à occuper tout ou partie de la résidence, la stèle fut sans doute

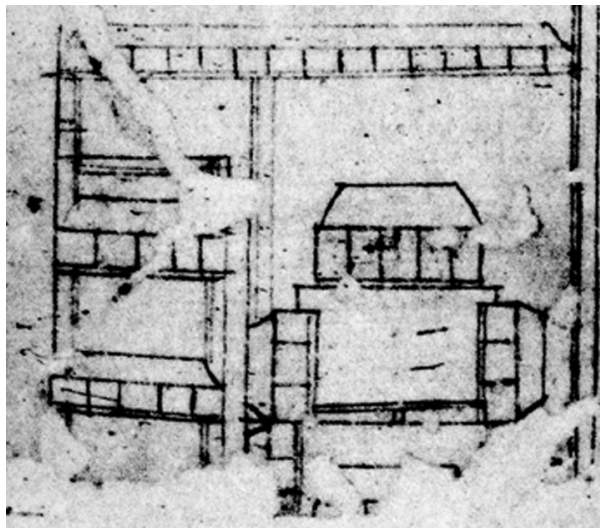
transportée au Miaoyuan guan, qui, entre temps, rapporte l'inscription de 1756 , avait été restauré par les princes du sang Zhuang qinwang 莊親王 et He qinwang 和親王 ${ }^{22}$ et confié à son tour à l'administration de Lou Jinyuan ${ }^{\varsigma 3}$.

Taishan xinyang beike jilu 北京東岳廟與北京泰山信仰碑刻紀錄, édité par Zhao Shiyu 趙世瑜 (Pékin, Zhongguo shudian, 2004), p. I53-154 et I68-170.

50. Il ne reste rien de ce site occupé aujourd'hui par les immeubles d'habitation de la Troupe nationale de théâtre (Zhongguo guojia huajuyuan).

5I. Da Zhenren fu bei, op. cit.

52. Heshuo Zhuang qinwang 和碩莊親王, Yinlu 㻆錄, était le seizième fils de Kangxi 康熙 (r. I662-1722); Heshuo He qinwang 和碩和親王, Hongzhou 弘昼, le cinquième fils de Yongzheng et le demi-frère de Qianlong. Ces deux princes patronnèrent l'édition de plusieurs ouvrages, notamment celle du Canon bouddhique de l'ère Qianlong (Qianlong dazang jing 乾隆大藏經) et un Index du Tripitaka (Daqing sanzang shengiiao mulu 大清三藏聖教目錄).

53. Miaoyuan guan bei, \# 543 (1756), BJTB, vol. 71, p. 74. 
La seconde stèle associant Lou Jinyuan et le Miaoyuan guan fut rédigée par un certain Shen Deqian 沈德潛 (I673-I769), poète et littérateur originaire de Suzhou. Reçu aux examens impériaux à l'âge de 66 ans, après dix-sept tentatives, Shen Deqian fit pourtant une belle carrière, d'abord à l'académie Hanlin, puis comme précepteur des princes impériaux, avant d'être nommé, à titre honoraire, ministre des Rites et grand précepteur du prince héritier. Après sa retraite, il devint en I75I recteur de l'académie Ziyang 紫陽 de Suzhou ${ }^{54}$. Alors qu'il est encore en poste à Pékin, il rend visite à un condisciple et payse venu à la capitale tenter sa chance aux examens mandarinaux ${ }^{55}$. Comme c'est souvent le cas des candidats peu fortunés, ce camarade loge dans un temple, et ce temple est le Miaoyuan guan. Shen Deqian remarque les stèles des Ming, et s'enquérant du maître des lieux, apprend qu'il s'agit de Lou Jinyuan. Il ne l'a jamais rencontré mais les deux hommes se connaissent du fait que le bureau de l'Administration du clergé taoïste, dont Lou Jinyuan est le directeur, dépend directement du ministère des Rites auquel le lettré est rattaché. Shen Deqian déplore que leurs occupations ne leur aient jamais ménagé le loisir de s'entretenir des «fondements de la quiétude et du non-agir » (tan qingiing wuwei aozbi 談清淨無為奧旨). Leur relation dut être suffisamment étroite pour que Lou Jinyuan, lorsqu'il envoya ses vœux à Shen Deqian qui fêtait ses 80 ans, le priât de rédiger l'inscription qui nous a été conservée. Shen Deqian y retrace aussi bien sa propre carrière que celle de Lou Jinyuan et évoque brièvement l'histoire du lieu et les mérites de ses bienfaiteurs ${ }^{56}$.

Cette inscription réunit plusieurs noms de l'élite aristocratique, littéraire et artistique de la capitale. Les princes Zhuang 莊 et $\mathrm{He}$ 和, le lettré Shen Deqian, mais aussi le célèbre peintre et calligraphe Dong Bangda 董邦達, qui a tracé les caractères du cintre de la stèle, ainsi que le prince Shen 慎, poète et calligraphe, qui calligraphia l'inscription elle-même ${ }^{57}$. Cet aréopage de princes et de lettrés, tous liés à un titre ou à un autre à la restauration du temple des Affinités merveilleuses, témoigne du prestige dont jouissait alors le sanctuaire, prestige accru par la réputation de son administrateur, Lou Jinyuan, le plus puissant maître taoïste du moment.

\section{Le déclin et la ruine}

À partir du milieu du XVIII ${ }^{\mathrm{e}}$ siècle, et pendant plus d'un siècle, le temple n'est plus guère documenté. Lorsqu'on retrouve sa trace dans les dossiers des Archives

54. Voir Arthur W. Hummel, Eminent Chinese of the Ch'ing Period, Taipei, SMC Publishing, 2002, vol. 2, p. $645^{-6} 646$.

55. La visite se situe entre 1746 , après que le temple eut été restauré et son administration confiée à Lou Jinyuan, et le printemps de l'année i75I, lorsque Shen Deqian retourna à Suzhou. Peut-être en 1749, par déduction du fait que des examens eurent lieu en 1752 et que les sessions étaient organisées tous les trois ans (Qingshi gao, 清史稿, II, p. 4I4 note en I75I que la prochaine session aura lieu "l'année suivante au deuxième mois »). Je remercie Guan Xiaojing 關笑晶 qui m’a aidée à établir cette chronologie.

56. Cette inscription a été étudiée dans le groupe de lecture évoqué plus haut.

57. Le prince Shen était Yunxi 允禧, 2 $\mathrm{I}^{\mathrm{e}}$ fils de Kangxi. 
municipales de Pékin 北京市檔案館, sa fortune est sur le point de s'éteindre. Ces archives ont conservé les documents issus de la procédure d'enregistrement des temples de la capitale conduite par deux organismes du gouvernement républicain. Les documents sont classés en trois séries correspondant à trois campagnes. La première série regroupe les documents réunis par le bureau de la Sûreté de la ville de Pékin 北平特 別市政府公安局 entre 1928 et I929; la seconde, ceux du bureau des Affaires sociales 北平市社 會局 entre I930 et 1945, et la troisième, compilée par le même bureau, n'est en fait qu'une liste des temples et de leurs responsables entre $1945^{-}$ 1946. Les deux premières séries de documents rassemblent les formulaires et les reçus d'enregistrement des temples, les certificats des garants - des commerçants voisins du sanctuaire, qui se portent garants de sa gestion régulière et de l'acquittement des impôts ; souvent les procèsverbaux des entretiens entre les fonctionnairesenquêteurs et le responsable du temple sont

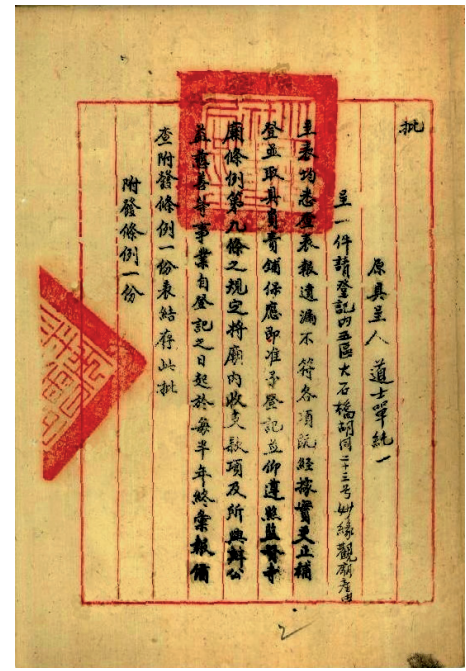

Fig. 7 : Page d'archive comportant la déclaration manuscrite du prêtre Shan Chunyi relative à l'enregistrement du Miaoyuan guan (liasse J2-8555 , p. 2). conservés (fig. 7). Ils concernent la plupart du temps la vérification des déclarations faites par les religieux à demeure, relatives tantôt au nombre de pièces, de moines résidents, de statues ou d'objets rituels, tantôt aux modalités de la transmission des titres de propriété et la régularité de la succession.

On trouve dans les archives républicaines les noms des trois prêtres taoïstes qui se transmirent la responsabilité du Miaoyuan guan entre i888 et $1922^{58}$. En I888, le prêtre taoïste qui en reçut la gestion était originaire du Jiangxi, tandis que le précédent responsable, qui venait de mourir, portait le titre de faguan 法官 ou « officier des rites ». Dans ce contexte, le titre de faguan désignait les prêtres taö̈stes de l'Église des maitres célestes qui occupaient une position officielle et avaient été nommés par la cour ou par l'administration du Longhushan dans les temples de la capitale ${ }^{59}$. La qualité de faguan du responsable du temple jusqu'en I888, et le lieu d'origine de son successeur - le Jiangxi où résidaient le Maitre céleste et son clergé - laissent supposer que le temple des Affinités merveilleuses était encore à cette époque affilié au Longhushan et qu'il recevait peut-être des subsides de la cour.

Le temple subit certainement des dommages pendant l'insurrection des Boxers en I900, puisqu'il est rapporté que son titre de propriété fut perdu durant les événements et dut être remplacé en 1903. En 1922, un nouveau prêtre nommé Shan Chunyi 單純一, originaire de Shaoxing 紹興 dans le Zhejiang 浙江, devint l'administrateur du temple. Comme nombre d'autres fondations religieuses, le Miaoyuan guan louait

58. Il s'agit de Huang Baoshou 黃保壽, Liu Juxian 劉巨铣 et Shan Chunyi 单純一.

59. Sur le titre de faguan, voir Vincent Goossaert, «Bureaucratic Charisma », p. I27. 
une partie de ses bâtiments à des particuliers qui, le plus souvent, y installaient des ateliers ou des dépôts de marchandises. Le dialogue conservé dans les Archives municipales de 1935 entre les inspecteurs du bureau des Affaires sociales et le prêtre Shan Chunyi témoigne de cette situation :

Question : Avez-vous retrouvé les statues en bronze?

Réponse : Je les ai retrouvées. Il y en a six, des grandes et des petites.

Question: Où les avez-vous trouvées?

Réponse : Comme nous louons une partie du temple à une papeterie, ils y entreposent du papier ; ils avaient entassé du rebut sur les statues.

Question : Maintenant que vous avez retrouvé les statues, il faut les porter à l'inventaire.

Réponse : Je vous prie de bien vouloir les rajouter ${ }^{60}$.

問：你尋找銅佛像情形如何?

答：業經找著, 共計大小六尊。

問 : 你在何處尋找?

答：在殿內找著。因本廟殿房租給染紙坊, 殿内存紙, 他們把銅像壓在爛紙底下。

問：既將銅像找著, 應補填表內。

答：請予補填。

L'inventaire systématique des temples de Pékin conduit entre I93I et I933 par l'Institut national de recherche de Beiping 國立北平研究院 est plus précis ${ }^{61}$. Non seulement il localise et recopie les cinq stèles encore sur place au moment de l'enquête - curieusement, il n'est pas question de la stèle « cartulaire » de $145 \mathrm{I}$ - mais il décrit les bâtiments et le mobilier liturgique. Malheureusement, contrairement à l'ordinaire, le dossier du Miaoyuan guan ne comprend pas de plan des lieux. Les enquêteurs remarquent en entrant dans le sanctuaire, encastrée au-dessus de l'entrée, une plaque de pierre portant l'inscription : «Antique temple Miaoyuan guan fondé par faveur impériale » 敕賜古妙緣觀 calligraphiée par l'empereur Chongzhen 崇禎 (r. I628-I644), le dernier souverain des Ming. Le temple était donc encore soutenu par la cour à la fin des Ming. À leur habitude, les enquêteurs consignent les objets précieux. Ils en mentionnent $\operatorname{six}^{62}:$ dans la première cour, un grand brûle-parfum en fer de I7I3 ; dans la seconde cour, à l'est deux «bol sonores » en fer tieqing 鐵馨, fabriqués en I675, l'un destiné à être « éternellement conservé » dans la salle des 《 ancêtres du Dao » (Daozu dian 道祖殿), c'est-à-dire celle des Trois Purs Sanqing 三清, et l'autre dans celle de Huangdi 黃帝 $^{63}$; devant la salle consacrée à Zhenwu et d'autres divinités secondaires ${ }^{64}$, est suspendue une cloche en bronze fondue en 1713 et un muyu 木魚; un brûle-parfum en fer de l'époque Qianlong, sans date, clôt la liste. Les enquêteurs remarquent encore deux vénérables acacias. Parmi les statues, ils notent une statue en bois de Doumu 斗母 dans la salle principale de la cour de l'est. Elle mesure près de deux mètres ; elle est placée sur une haute estrade et accompagnée des Seigneurs stellaires des constellations des trois palais (dong xi zhong

60. Archives municipales de Pékin, liasse J2-8-555, p. 35.

6I. Archives du Beiping yanjiu yuan, dossier «Miaoyuan guan », Xisi 西四 4.

62. Les Archives municipales qui font un inventaire complet en inscrivent dix-neuf.

63. Il s'agit en réalité de Yuhuang dadi 玉皇大帝 dont la statue figure à l'inventaire des Archives municipales de 1936 (J2-8-555, p. 53).

64. Le rapport parle de pusa 菩薩 et de daoren 道人 pour désigner ces statues non identifiées. 
dou sanfu xingjun 東西中斗三府星君), représentés par des statues en bois de plus petite taille, et de quatre jeunes serviteurs (tongxiang 童像). L'enquêteur mentionne encore les statues des maîtres des trois doctrines, Confucius, Bouddha et Laozi, et celle de Huangdi (c.-à-d. Yuhuang), accompagnée de six autres petites statues en bois.

Des statues, des brûle-parfums, des cloches et de tout le riche mobilier du temple, rien ne subsiste aujourd'hui, pas même les arbres.

A la veille de l'occupation japonaise, le Miaoyuan guan avait définitivement perdu le lustre d'antan. Seule l'une des grandes salles était encore consacrée au culte, le reste du temple était loué. De plus, lorsqu'un des bâtiments latéraux fut démoli en 1936, les matériaux servirent à réparer celui situé en vis-à-vis, un procédé bien éloigné des riches aménagements d'autrefois. Dans les années 1940, une nonne taoïste habitait la cour intérieure, tandis qu'un couple de cordonniers, qui faisait office de gardien, habitait à l'entrée du temple. Une fonderie occupa les lieux dans les années soixante. En 2006, il ne restait que quelques dépendances de l'ancien temple utilisées par une fabrique de cartonnage. Les gens du voisinage se souvenaient de la grande salle principale, celle dans laquelle on venait encore brûler de l'encens et prier. Elle fut rachetée par un «capitaliste » qui en fit sa résidence, probablement jusque dans les années I950, avant qu'elle ne devienne avec les autres bâtiments propriété de l'État.

Même lacunaire, l'histoire du temple des Affinités merveilleuses, telle qu'elle vient d'être exposée, s'étend sur près de six cents ans. Fondation taoïste dès sa construction par un eunuque vietnamien, le Miaoyuan guan reçut le soutien de la cour et des plus hauts représentants de l'État. Il le conserva jusqu'au milieu du $\mathrm{XVIII}^{\mathrm{e}}$ siècle, lorsqu'il devint l'une des résidences du puissant représentant du clergé taoïste Lou Jinyuan. Sa restauration se fit sous l'égide de l'aristocratie mandchoue et des élites lettrées. À la chute de l'empire — ou avant déjà — le temple, privé du soutien de ses patrons fortunés et des subsides impériaux, amorça sa période de déclin ; aucune communauté de fidèles ne semble avoir relayé le soutien des élites et maintenu le temple en activité. Du moins, les documents réunis jusqu'à ce jour n'en ont pas conservé le témoignage. 